\title{
Land evaluation, characterization and classification of soil for the proposed oil palm plantation in Ekpri Ibami, Akamkpa Local Government Area, Nigeria.
}

\author{
Kingsley John ${ }^{1 *}$, Ackley Ufot Akpan-Idiok ${ }^{2}$ \\ (CULS) Kamýcká 129, 16500 Praha 6-Suchdol. \\ ${ }^{2}$ Department of Soil Science, University of Calabar, PMB 1115, Nigeria. \\ *Corresponding Author
}

${ }^{1}$ Department of Soil Science, Faculty of Agrobiology, Food and Natural Resources, Czech University of Life Sciences Prague

\begin{abstract}
Land evaluation, characterization and classification of soil for the proposed oil palm plantation in Ekpri Ibami, Akamkpa Local Government Area, Nigeria was conducted on 50 ha of land using a combination of both conventional and digital survey methods. The objective of the research was to characterize, classify and evaluate the land for the proposed oil palm production. Three soil mapping units were identified (EKP I, EKP II and EKP III) and representative profile pits were dug in each mapping unit. Samples were collected from each pedogenic horizon. Soils morphological, physical and chemical properties were determined using appropriate methods. Results revealed that soils of all of the mapping are characterized by dark greyish brown colour with thin topsoil while yellowish red to strong brown mottles were observed at the subsoil. The soils are coarsed-textured with high sand, low silt, and clay fractions. The soils were strong to moderately acidic (4.8 to 5.3). The soils also have low inherent of natural fertility with low organic carbon content, total nitrogen, and moderately available phosphorus. Low effective cation exchange capacity and high base saturation which may have occurred in available forms in solutions in spite of the low cation reserves in the soil. The individual mapping units were classified as Arenic Eutrudept, Typic Hapludult and Aquic Paleudults for EKP I, EKP II and EKP III respectively. The land was then evaluated and classified based on its suitability for oil palm production. Parametrically, mapping units EKP I and EKP II were marginally suitable and EKP III not suitable for the proposed oil palm production. Non-parametrically, EKPI is moderately suitable, EKP II is marginally suitable and EKP III is not suitable, for growing oil palm. The prevailing limitations on the Ekpri Ibami landscape for oil palm
\end{abstract}

production include fertility, wetness, and topography and soil physical properties. Fertility factor happened to be the most limiting factor in all the mapping units. And thus can be ameliorated through the application of organic manures, NPK fertilizer and liming to improve the nutrient status of the soil.

Keywords- Land evaluation, characterization, classification, sustainable agriculture.

\section{INTRODUCTION}

Ekpri Ibami is an agrarian community where dwellers are mainly involved in arable and tree crops production, especially Oil palm with less emphasis on fishing, hunting, and mining of solid minerals. Despite the high agricultural potential of the area, there has been known little or no published land evaluation and soil characterization articles to make sure easy access and transfer of technology to areas with similar soils elsewhere. Due to the little or no information on land evaluation and soil studies in the location, the farmers are often compelled to grow their crops without adequate attention as a result,soils are not used for the purpose that best suits their properties.Land evaluation,however,approaches targeted towards sustainable agriculture and happens to be the starting point towards adequate information on land resources, which land evaluation,provides during soil characterization studies. In this process, soil resources must be studied in detail through processes of soil characterization and land ratings for the land utilization under consideration (Esu, 2004); such information highlights soil characteristics and conditions that are suitable for growing specific crops (Ogunkunle, 2005). It is therefore apt to evaluate soil mapping units obtained from a 
soil survey for Oil palm cultivation. Furthermore, adequate information is often generated during soil surveys to "determine the important characteristics of soils,classify them into mapping units,set up and plot on maps the boundaries between kinds of soils and to correlate and predict the adaptability of soils to various crops".

The earlier reporton the soils of Ekpri Ibami have revealedwell-drained soils with coarse-textured,strongly acid reaction,moderate organic carbon, and nitrogen but low in available $\mathrm{P}$ and basic cations with quartz, kaolinite, and microcline as dominant minerals (Aki et al., 2014). A similar report by Abua and Eyo (2013)of the same study revealed a coarse-textured in the surface horizonwith subsurface accumulation of clay and $\mathrm{pH}\left(\mathrm{H}_{2} \mathrm{O}\right)$ range of 5.46.8, but moderate organic carbon and total nitrogen,low available $\mathrm{P}$ and exchangeable bases. But, $\mathrm{pH}$ value of 3.8 was obtained by Attoe et al. (2016) in the Basement complex soils of Akamkpa with moderate organic carbon and total nitrogen but low available $\mathrm{P}$ and exchangeable cations as obtained by Aki et al., (2014), and Abua and Eyo (2013). The soils,so, have high agricultural potentials but may needadequate soil management strategies.

Owing to the basic principles of conventional soil mapping,digital soil mapping (DSM) techniques, a world trending tool was adopted in this investigation.Digital soil mappingis a computer-assisted production of digital maps of soil types and properties. The method employsthe application of mathematical and statistical models that corroborate information from soil observation with information contained in correlated environmental variables (Dobos et al., 2006) and uses GIS and computer programming to put into a quantitative framework the study of soils (McBratney et al., 2003; Mckenkie and Ryan, 1999).

Oil palm production, which the soils are were investigated for, is one of the most important tree crops that have almost none of its parts as a waste. Its numerous importance has endeared the Federal Government of Nigeria to select it as a value chain crop; also, its cultivation and processing requires little skills and so can be cultivated by rural farmers with little supervision. This is probably the reason why most farmers in the locality grow the crop in either small or large scale. It is proposed to set up an Oil Palm estate at Ekpri Ibami thereby creating jobs for the dwellers.The study was aimed at evaluation, characterization, and classification of soil for the proposed
Oil Palm plantation in Ekpri Ibami,Akampa Local Government Area of Cross River State.

\section{$2.1 \quad$ Location of the Study}

The study was conducted on Ekpri Ibami landscape on the coordinates $\left(05^{\circ} 18^{\prime} 53^{\prime \prime} \mathrm{N}, 08^{\circ} 13^{\prime} 25^{\prime \prime} \mathrm{E}\right.$; 82111 m ASL) within Akamkpa Local Government Area of southern Cross River State (Fig. 1). The area is within the humid tropical climate characterized by distinct wet and dry seasons. The mean annual rainfall ranges between 1500$3500 \mathrm{~mm}$, relative humidity $80-90 \%$ and mean annual temperature value between $25.4-27.5^{\circ} \mathrm{C}$ (NIMET, 2015). These datawere adapted from Calabar weather station of the Nigerian Meteorological Agency being situated within 100 $\mathrm{km}$ range of thesynoptic station as proposedby Afangide $e t$ al.(2010) (Table 1).

The location of the study is on a basement complex geological material of which $40 \%$ encapsulates the entire southern Cross Rivers State, Nigeriaspreading up to the African-pan basement complex of Cameroun highlands. The characteristics of the material showthe processes that form the underlying and the influence of the environment where which they occurred.

\subsection{Vegetation and land use}

The area is covered with secondary forest regrowth with few annual crops identified consist of Zea mays, Manihot spp, Oryza sativa, Musa spp, Dioscorea spp, and perennial crops such as Carica papaya, Elaeis guineensis, Hevea brasiliensisand Irvingia gabonensis. Dominant trees, climbers, and shrubs such as Daniella oliveri, Ficus spp, Khaya senegalensis,Laxifora spp, Combretum spp,Alcohornea spp, Andropogon spp, and Digitaria spp, are scattered almost evenly while African bamboo trees grow wildly near the streams and lowland areas.

\section{$2.3 \quad$ Field Study}

The research was conducted on a 50-hectare land. A semidetailed survey was employed at a scale of 1:50 000 which enabled delineation of the boundary through the acquisition of (x), longitude (y) and elevation (z) [xyz]values with the aid of a German Etrex (2000) global positioning system (GPS) device.The xyz values were used to obtain the topographic sheet of the location. Digital terrain model (DTM) of the study area was created from the topographic map; slope map was extracted from the DTM which was classified into different categories. 


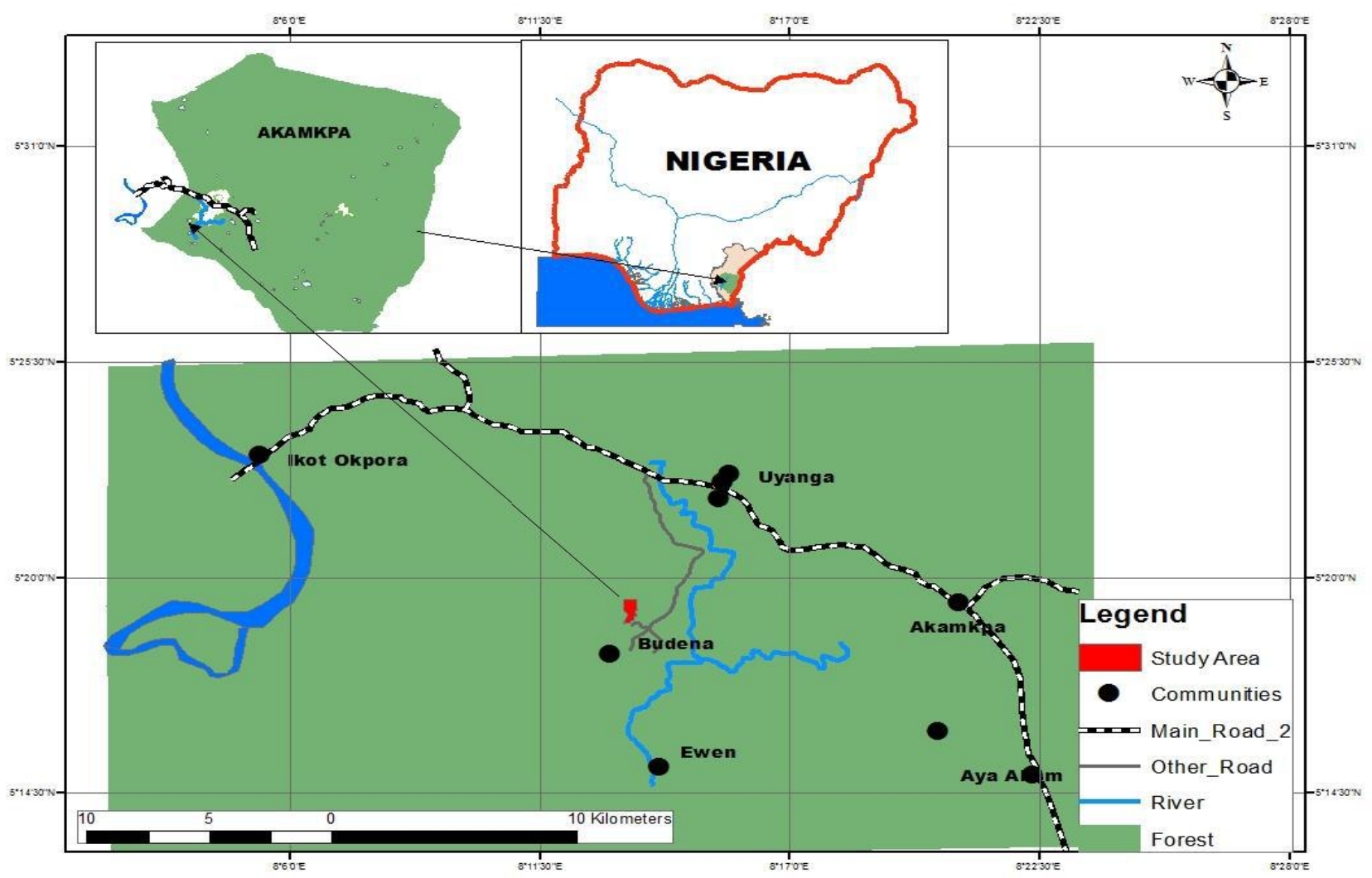

Fig.1: Location Map of the Study Area

Table.1: The Mean annual rainfall, humidity, temperature regime and sunshine duration between 1997 and 2014 in Calabar,

Cross River State, Nigeria

\begin{tabular}{llll}
\hline Year & $\begin{array}{l}\text { Rainfall } \\
(\mathbf{m m})\end{array}$ & $\begin{array}{l}\text { Dry-bulb air } \\
\text { temperature }\left({ }^{\mathbf{}} \mathbf{C}\right)\end{array}$ & $\begin{array}{l}\text { Relative } \\
\text { Humidity }(\boldsymbol{\%})\end{array}$ \\
\hline 1997 & 3282.9 & 26.9 & 84.8 \\
1998 & 2512.6 & 27.6 & 83.3 \\
1999 & 2673.1 & 27.5 & 84.7 \\
2000 & 2678.6 & 27.2 & 85.1 \\
2001 & 3073.6 & 26.9 & 82.9 \\
2002 & 2691.4 & 26.7 & 83.3 \\
2003 & 2113.7 & 27.5 & 83.3 \\
2004 & 1729.1 & 27.5 & 85.2 \\
2005 & 2583.3 & 27.3 & 82.7 \\
2006 & 2951.8 & 26.9 & 84.2 \\
2007 & 2417.5 & 26.1 & 84.6 \\
2008 & 1931.3 & 26.7 & 83.7 \\
2009 & 1401.1 & 26.7 & 81.8 \\
2010 & 2003.7 & 26.8 & 82 \\
2011 & 2969.8 & 26.1 & 82.5 \\
2012 & 4364.1 & 27.2 & 86.3 \\
2013 & 3506.4 & 26.9 & 86.4 \\
2014 & 3433.3 & 25.4 & 86.3 \\
Mean & $\mathbf{2 6 8 4 . 3}$ & $\mathbf{2 6 . 9}$ & $\mathbf{8 4 . 1}$ \\
\hline
\end{tabular}

Source: National Meteorological station (NIMET) Calabar (2015) 
Each slope category became a soil mapping unit.A systematic special purpose grid system detailed soil survey was adopted for ground-truthing of the computer delineated mapping units to establish the actual soil boundaries on the field. Rigid auguring at $50 \mathrm{~m}$ x $50 \mathrm{~m}$ intervals was made, physical and morphological properties were recorded for use in demarcating the mapping unit's boundaries.

\subsection{Soil sampling and preparation}

Three (3) mapping units, EKP I, EKP II and EKP III were identified. A pedon was cited on each mapping unit to the depth of $200 \mathrm{~cm}$ except interfered by an impenetrable layer or water table. The soils were described according to the guidelines of USDA-NRCS (Soil Survey Staff, 2002) as modified by Ibanga (2003) and Esu (2010). Undisturbed core cylinder samples were collected for bulk density and porosity determinations. Samples were then taken from each pedogenic horizon, bagged, labelled and transported to the laboratory for analysis. Prior to analysis, the samples were air dried, gently crushed and sieved through $2 \mathrm{~mm}$ sieve.

\subsubsection{Laboratory analysis}

The particle size distribution was determined using the Bouyoucos hydrometer method (Gee and Bauder, 1986) and the percent sizes later used to ascertain the soil textural class with the aid of the soil textural triangle provided by USDA. Soil $\mathrm{pH}$ was determined potentiometrically in the soil-water ratio of 1:1 (Udo et al., 2009) and organic carbon was determined by the Walkley and Black wet oxidation method. Exchangeable cations were extracted using $1 \mathrm{~N}$ $\mathrm{NH}_{4} \mathrm{OAc}$ ( $\mathrm{pH}$ 7.0) and, exchangeable $\mathrm{Ca}^{2+}$ and $\mathrm{Mg}^{2+}$ determined by atomic absorption spectrophotometry (Thomas, 1982) while $\mathrm{Na}^{+}$and $\mathrm{K}^{+}$were determined by flame photometry. Effective cation exchangeable capacity was determined by summing up the exchangeable bases and exchangeable $\mathrm{Al}^{3+}$ as outlined by Udo et al (2009). Base saturation was obtained by expressing the sum of exchangeable cations as a percentage of the cations exchange capacity (IITA, 2000) using the formula; B.S = $\left[\frac{T E B}{E C E C}\right] \times 100(\%)$.

\subsection{Soil Classification}

The pedons were classified based on USDA Soil Taxonomy (Soil Survey Staff, 2010). The Soil Taxonomy was based on the properties of the soil as were found during the study. The physical, chemical and morphological properties and general site information were used as criteria to clas sify the soil to the subgroup level.

\subsection{Land evaluation}

The land evaluation was carried out according to the guidelines provided by Sys (1985) (Table 2). This is to make sure the reduction in the risk of production through the matching of the requirements of land use to the land qualities. Three (3) soil units were placed in the suitability classes by comparing the data obtained in the area under study for oil palm production to Oil palm requirement information. The evaluation was both conventional (nonparametric) (FAO, 1976) and parametric methods (Ogunkunle, 1993; Udoh et al., 2006). For the nonparametric evaluation, pedons were first placed in suitability classes by matching their characteristics with the established requirements. The aggregate suitability classes were indicated by the most limiting characterist (c(s) of the soil units. For the parametric method, each characteristic was rated and the index of productivity (IP) for each pedon was calculated using the square root method equation:

$$
\mathrm{IP}=A x \sqrt{B} / 100 x C / 100 x \ldots x F / 100
$$

Where: $\mathrm{A}$ is the overall lowest characteristic rating and $\mathrm{B}, \mathrm{C}, \ldots \mathrm{F}$ is the lowest characteristic ratings for each land quality group (Udoh et al., 2006). "Five land quality groups climate (c), topography (t), soil physical properties (s), wetness (w) and fertility (f) were used in this method of evaluation" (Table 2). "Only one member characteristics in each land quality group will be used for calculation purpose because there are usually strong correlations among members of the same group" (Ofem et al., 2016). "Five levels of limitations were used, no limitation (0), slight limitation (1), moderate limitation (2) severelimitation (3) and very severe limitation (4)" (Table 3). One "limitation level was attributed to each land characteristic. The final (aggregate) suitability classes were determined by the number and intensity of the limitation(s)", and the most unfavourable characteristic determined the suitability classification. Suitability classes S1, S2, S3, N2, and N1 were established as reported by Ofem et al (2016). For actual (current) productivity index, all the lowest characteristic ratings for each land quality group were substituted into the index of productivity equation above. But, in the case of potential productivity index, it was assumed that the corrective fertility measure would no longer have fertility constraints. So, other qualities except for fertility (f) were used to calculate the potential productivity index. "Suitability classes S1, S2, S3, and N are equivalent to IP values of $100-75,74-50,49-25$ and $24-0$, respectively".

\section{RESULTS AND DISCUSSION}

\subsection{Morphological and physical properties:}

Threesoil mapping units were identified in the study area denoted by EKP I, EKP II and EKP III.

Soil mapping unit EKP I: The soils are very deep (>100 $\mathrm{cm})$, located at the crest region of the landscape with an elevation value of $108 \mathrm{~m}$ above sea level (Table 4). The Ap 
horizon of the soil was observed to be thin $(16-17 \mathrm{~cm})$ with an extensive thick B-horizon which may be attributed to eluviation-illuviation processes (FitzPatrick, 1986; Orimoloye et al., 2010). The soil were very dark greyish brown (10YR 3/2) with no mottles at surface while the subsurface soils varied from yellowish red (5YR 5/8) ta reddishsh colour (2.5YR 4/6) with mottles occurring within the depths which may be attributed to periods of wetting which had led to inadequate aeration or reduction during the periods of the year, leading to oxidation-reduction reactions. The soils are weakly structured and there is evidence of layers with weak pedogenic processes in the field. The soils are coarse-textured with high sand content $=623.5 \mathrm{~g} / \mathrm{kg}$, low silt $=99.0 \mathrm{~g} / \mathrm{kg}$ and low clay $=277.5 \mathrm{~g} / \mathrm{kg}$ and a sandy loam texture in the surface horizon and sandy clay loam in the subsurface horizon. The high silt/clay ratio indicates a high intensity of weathering (Young, 1976). Bulk density values varied from $1.2 \mathrm{~g} / \mathrm{cm}^{3}$ at the surface horizon to 1.5 $\mathrm{g} / \mathrm{cm}^{3}$ in the subsurface horizon (Table 5). These values will allow root penetration, and good aeration, water movement for best crop production (Esu, 2010).

Table.2: Modified Land Suitability Requirements for Oil Palm Based on Land Characteristics

\begin{tabular}{|c|c|c|c|c|c|}
\hline \multirow[t]{2}{*}{ Parameters } & \multicolumn{5}{|c|}{ Suitability class } \\
\hline & S1 & S2 & $\mathbf{S 3}$ & N1 & $\mathbf{N 2}$ \\
\hline Land Qualities & $100-75$ & $74-50$ & $49-25$ & $24-15$ & $14-0$ \\
\hline \multicolumn{6}{|l|}{ Climate (c) } \\
\hline Annual Rainfall (mm) & $>1700$ & $1450-1700$ & $1250-1450$ & - & $<1250$ \\
\hline $\operatorname{MAT}\left({ }^{\circ} \mathrm{C}\right)$ & $>22$ & $20-22$ & $18-20$ & - & $<18$ \\
\hline Relative humidity (\%) & $>70$ & $65-70$ & $60-65$ & - & $<60$ \\
\hline \multicolumn{6}{|l|}{ Topography(t) } \\
\hline Slope $(\%)$ & $0-8$ & $8-16$ & $16-30$ & $>30$ & - \\
\hline \multicolumn{6}{|l|}{ Wetness (w) } \\
\hline Flooding & Fo & $\mathrm{F} 1$ & $\mathrm{~F} 2$ & - & F3 \\
\hline Drainage & Perfect, well & Mod. Well & Poor, aeric & Poor, drainable & $\begin{array}{l}\text { V. poor, not } \\
\text { drainable }\end{array}$ \\
\hline \multicolumn{6}{|c|}{ Soil Physical properties(s) } \\
\hline Texture & $\mathrm{Cl}, \mathrm{Scl}, \mathrm{L}$ & Scl & Scl-Lfs & Any & $\mathrm{C}, \mathrm{Cs}$ \\
\hline Structure & Blocky & - & - & - & $\begin{array}{l}\text { massive, single } \\
\text { grain }\end{array}$ \\
\hline Depth $(\mathrm{cm})$ & $>100$ & $50-100$ & $25-50$ & - & $<25$ \\
\hline \multicolumn{6}{|l|}{ Fertility(f) } \\
\hline ECEC $(\mathrm{cmol} / \mathrm{kg})$ & $>8.0$ & $5-8$ & $<5$ & - & - \\
\hline Base Sat. (\%) & $>35$ & $20-35$ & $<20$ & - & - \\
\hline $\mathrm{pH}\left(\mathrm{H}_{2} \mathrm{O}\right)$ & $5.5-6.0$ & $5.5-6.0$ & $6.5-7.0$ & $<4.0,>7.0$ & $<4.0,>7.0$ \\
\hline $\mathrm{OC}(\%)(0-15 \mathrm{~cm})$ & $>1.2$ & $1.2-0.5$ & $0.5-0.3$ & $0.3-0.2$ & $<0.2$ \\
\hline
\end{tabular}

Flooding: Fo, No flooding; F1, 1-2 flooding months in>10 yrs; F2, Not more than 2-3 months in 5 yrs out of 10 yrs; F3, 2-4 months almost every year; F4, >4 months in almost every. Texture: Cl, clay loam; Scl, Sandy clay loam; L, Loam; Lfs, Loamy fine sand; c, Clay; Cs, Clayey s and MAT= Mean annual temperature.

Source: Sys et al. (1991) 
Table.3: Ratings oflimiting characteristics.

\begin{tabular}{ll}
\hline Limitation & Rating \\
\hline Slight to none & $100-90$ \\
Slight & $89-70$ \\
Moderate & $69-50$ \\
Severe & $49-35$ \\
Very Severe & $34-0$ \\
Can be corrected & $34-20$ \\
Cannot be corrected & $19-0$ \\
\hline
\end{tabular}

Soil mapping unit EKP II: It is well deep $(>100 \mathrm{~cm})$ with sandy loam texture and they occupy the middle slope of the landscape under study at an elevation of $102 \mathrm{~m}$. The soil colour is characterized with a distinct dark yellowish brown (10 YR 4/4) at the surface horizon coming down to yellowish brown (10 YR 4/6) in the subsurface. Dark red (10 R 3/6) coloured mottles was observed in the subsurface layer and this may be as a result of poor aeration leading to oxidation-reduction reactions. Structural aggregates varied from weak medium granular in the surface and moderate medium coarse subangular blocky in the subsurface. Silt/clay ratio revealed that the soils possess high weathering potentials $(>0.15)$. Bulk density varied from 1.1 $\mathrm{g} / \mathrm{cm}^{3}$ in the surface horizon to $1.5 \mathrm{~g} / \mathrm{cm}^{3}$ in the subsurface horizon. These values obtained have been reported to be suitable for agricultural purposes (Esu, 2010).

Soil mapping unit EKP III: It covered the lower slope of the landscape and situated at the elevation of $82 \mathrm{~m}$ above sea level. The soils are poorly drained with depth $<50 \mathrm{~cm}$. The hue values were $10 \mathrm{YR}$ and $7.5 \mathrm{YR}$ in the surface and subsurface layers giving a variation of colour, very dark grey-brown and strong brown, this result is similar with the report by Dengiz et al. (2012). Also, there was the occurrence of yellowish red (5 YR 4/6) mottle in the subsurface layer which indicates poor drainage conditions. Structural aggregates varied from moderate medium subangular blocky to weak fine granular in the surface and from moderate fine subangular blocky to weak fine medium granular in the subsurface horizon. The soils are predominantly sandy loam texture with particle size distribution $(\mathrm{sand}=703.2 \mathrm{~g} / \mathrm{kg}$, silt=102.9 g/ $\mathrm{kg}$ and clay= $203 \mathrm{~g}$ ). Silt/clay ratio revealed that the soils have high weathering potential $(>0.15)$. Bulk density values ranged from $1.1 \mathrm{~g} / \mathrm{cm}^{3}$ in the surface horizon to $1.5 \mathrm{~g} / \mathrm{cm}^{3}$ in the subsurface horizon.

\subsection{Chemical properties of the soil mapping units}

The chemical properties of the soil mapping unitsidentified on Ekpri Ibami landscape are presented in Table 6. The soil $\mathrm{pH}$ distribution in the soil units followed an irregular increase and decrease with depth. The mean $\mathrm{pH}$ $\left(\mathrm{H}_{2} \mathrm{O}\right)$ were 5.3, 4.8 and 4.9 in soil mapping unit EKP I, EKP II and EKP III, respectively. Negative delta $\mathrm{pH}(\Delta \mathrm{pH})$ obtained in the soils showed that all the layers of the soils profiles possessed net negative surface charges and such that they can retain basic nutrients for subsequent release into soil solution for plant uptake. Organic carbon and total nitrogen gave an irregular increase an a decreasese in values with depth. Means of organic carbon were $0.56 \%, 0.47 \%$ and $0.2 \%$ for EKP I, EKP II and EKP III respectively. While the means of total nitrogen content in the proposed land were $0.18 \%, 0.04 \%$ and $0.02 \%$ for EKP II and EKP III respectively. Organic carbon content and total nitrogen were rated low as they fall within thin the critical limit of $<1.5 \%$ and $<0.2 \%$ respectively (Enwezor et al.,1989). 


\begin{tabular}{|c|c|c|c|c|c|c|c|c|}
\hline Horizon & $\begin{array}{l}\text { Depth } \\
\text { (cm) }\end{array}$ & $\begin{array}{l}\text { Munsell- } \\
\text { colour } \\
\text { (moist) }\end{array}$ & Mottling & Texture & Structure & Consistence & Boundary & Other characteristics \\
\hline \multicolumn{9}{|c|}{${\mathrm{N} 05^{\circ}}^{19}{ }^{\prime} 16.84 " ; \mathrm{E008}^{\circ} 13 ' 36.1 " ; 108 \mathrm{~m} \mathrm{ASL}$} \\
\hline Ap & $0-17$ & $\begin{array}{l}\text { 10YR } 3 / 2 \\
\text { vdgb }\end{array}$ & & SL & $1 \mathrm{mcgr}$ & w ss, f, p & $\mathrm{cs}$ & $\begin{array}{l}\text { Porous, many fine medium roots, } \\
\text { Common medium roots; Iron concretion, } \\
\text { animal faecal, termite hill, quartzite } \\
\text { inclusion. }\end{array}$ \\
\hline Bw1 & $17-62$ & $5 \mathrm{YR} 5 / 8, \mathrm{yr}$ & & $\mathrm{scl}$ & $1 \mathrm{msbk}$ & wss, $\mathrm{f}, \mathrm{p}$ & gs & $\begin{array}{l}\text { Many medium pores, many common fine } \\
\text { medium roots, weathered rock mica } \\
\text { flakes, quartz inclusion, termites, } \\
\text { earthworms and ants activities. }\end{array}$ \\
\hline Bw2 & $62-122$ & $5 \mathrm{YR} 5 / 8, \mathrm{yr}$ & $\begin{array}{l}\mathrm{m}(10 \mathrm{R} 3 / 6 \\
\mathrm{dr})\end{array}$ & $\mathrm{scl}$ & $2 \mathrm{msbk}$ & w ss, f, p & ds & $\begin{array}{l}\text { Few medium pores, many common fine } \\
\text { medium roots, ants activities. }\end{array}$ \\
\hline Crt & $122-200$ & $2.5 \mathrm{YR} 4 / 6, \mathrm{r}$ & $\begin{array}{l}\mathrm{m}(10 \mathrm{R} 3 / 8 \\
\mathrm{yr})\end{array}$ & $\mathrm{scl}$ & $2 \mathrm{msbk}$ & wss,f, p & cs & $\begin{array}{l}\text { Many coarse pores, few medium roots, } \\
\text { ants activities clear, few thin cutans at ped } \\
\text { faces, many iron concretions. }\end{array}$ \\
\hline \multicolumn{9}{|c|}{ EKP IIN05 ${ }^{\circ} 1^{\prime} 11.3^{\prime \prime} ;$ E008 $^{\circ} 13^{\prime} 36.8^{\prime \prime} ; 102$ m ASL } \\
\hline Ap & $0-8$ & $\begin{array}{l}10 \mathrm{YR} 4 / 4, \\
\mathrm{dyb}\end{array}$ & & SL & $1 \mathrm{mgr}$ & ws s & $\operatorname{cs}$ & $\begin{array}{l}\text { Fine-coarse medium pores very fine size, } \\
\text { few medium roots fine size, } \\
\text { ants termites, earthworm and frogs, }\end{array}$ \\
\hline Bt1 & $8-53$ & $\begin{array}{l}10 \text { YR 4/4, } \\
\text { dyb }\end{array}$ & & SL & $2 \mathrm{mcsbk}$ & ws & $\mathrm{cd}$ & $\begin{array}{l}\text { Many medium pores, many common fine } \\
\text { medium roots, weathered rock }\end{array}$ \\
\hline Bt2 & $53-114$ & $10 \mathrm{R} 5 / 6, \mathrm{yb}$ & $10 \mathrm{R} \mathrm{3/6, \textrm {dr }}$ & SL & $2 \mathrm{msbk}$ & Wvs & gs & $\begin{array}{l}\text { mica flakes, quartz, termite activities. } \\
\text { Many fine and medium pores, fine } \\
\text { medium coarse roots, fine mica flake } \\
\text { and many ants. }\end{array}$ \\
\hline Crt & $114-200$ & $\begin{array}{l}10 \text { YR 5/6, } \\
y b\end{array}$ & $10 \mathrm{R} \mathrm{3/6, \textrm {dr }}$ & SL & $2 \mathrm{msbk}$ & Wvs & cs & $\begin{array}{l}\text { Fine medium coarse roots, few pores; fine } \\
\text { mica flakes. }\end{array}$ \\
\hline EKP III & \multicolumn{8}{|c|}{ 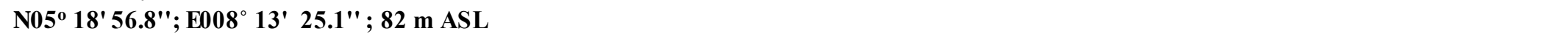 } \\
\hline Ap & $0-15$ & $\begin{array}{l}10 \mathrm{YR} 3 / 2 \\
\mathrm{vdgb}\end{array}$ & $\mathrm{nm}$ & $\mathrm{Sl}$ & $2 \mathrm{msbk}$ & wss, sp,f & $\operatorname{cs}$ & $\begin{array}{l}\text { Many medium pores, many fine roots, } \\
\text { many wormholes, termite activities } \\
\text { observed. }\end{array}$ \\
\hline Bt1 & $15-25$ & 7.5 YR 5/6, & $\mathrm{nm}$ & $\mathrm{Scl}$ & $2 \mathrm{fsbk}$ & wss, $\mathrm{p}, \mathrm{f}$ & $\mathrm{dw}$ & Few thin clay cutan at ped faces, many \\
\hline
\end{tabular}




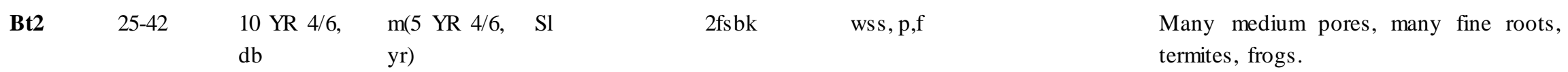

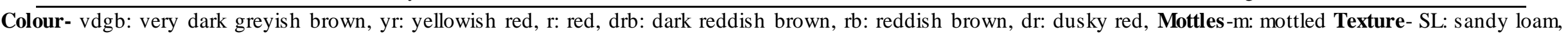

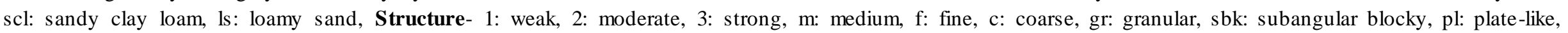
Consistence- wssfp: wet, slightly sticky, friable, plastic, ws sp: wet, slightly sticky, ws: wet, sticky, Boundary- cs: clear smooth, ASL: above sea level.

\begin{tabular}{|c|c|c|c|c|c|c|c|c|c|}
\hline \multirow[t]{2}{*}{ Horizon } & \multirow[t]{2}{*}{ Depth $(\mathrm{cm})$} & \multirow[b]{2}{*}{ Sand } & \multicolumn{2}{|c|}{$\begin{array}{l}\text { Particle Size Distribution } \\
\mathrm{gkg}^{-1}\end{array}$} & \multirow[t]{2}{*}{$\begin{array}{l}\text { Textural } \\
\text { Class }\end{array}$} & \multirow[t]{2}{*}{ Silt: Clay } & \multirow[t]{2}{*}{$\begin{array}{l}\text { Gravel } \\
\text { (\% vol) }\end{array}$} & \multirow{2}{*}{$\begin{array}{l}\text { Bulk } \\
\text { density } \\
\left(\mathrm{g} / \mathrm{m}^{3}\right)\end{array}$} & \multirow[t]{2}{*}{$\begin{array}{l}\text { Porosity } \\
(\%)\end{array}$} \\
\hline & & & Silt & Clay & & & & & \\
\hline \multicolumn{10}{|c|}{ EKP IN05 ${ }^{\circ} 19 ’ 16.84 " ;$ E008 $^{\circ}$ 13'36.1” ;108 m ASL } \\
\hline Ap & $0-17$ & 766.4 & 83.6 & 150 & SL & 0.56 & 7.7 & 1.2 & 55 \\
\hline Bw1 & $17-62$ & 614.8 & 65.2 & 320 & SCL & 0.20 & 11.5 & 1.5 & 43 \\
\hline Bw2 & $62-122$ & 616.4 & 103.6 & 280 & SCL & 0.37 & 64.8 & 1.5 & 43 \\
\hline $\mathrm{Crt}$ & $122-200$ & 496.4 & 143.6 & 360 & SCL & 0.40 & 63.7 & 1.4 & 47 \\
\hline Range & & $496.4-766.4$ & $65.2-143.6$ & $150-360$ & & $0.20-0.56$ & $7.7-64.8$ & $1.2-1.5$ & $43-55$ \\
\hline Mean & & 623.5 & 99.0 & 277.5 & & 0.3825 & 36.9 & 1.4 & 47 \\
\hline \multicolumn{10}{|c|}{ EKP IIN05 ${ }^{\circ} 19^{\prime} 11.3^{\prime \prime} ; \mathrm{E008}^{\circ} 13^{\prime} 36.8^{\prime \prime} ; 102 \mathrm{~m} \mathrm{ASL}$} \\
\hline Ap & $0-8$ & 635.2 & 134.8 & 230 & SL & 0.59 & 72.0 & 1.1 & 58 \\
\hline Bt1 & $8-53$ & 695.2 & 142.8 & 162SL & SL & 0.88 & 0.0 & 1.4 & 43 \\
\hline $\mathrm{Bt} 2$ & $53-114$ & 712.2 & 97.8 & 190SL & SL & 0.51 & 57.0 & 1.5 & 43 \\
\hline $\mathrm{Crt}$ & $114-200$ & 575.2 & 44.8 & 380 & SL & 0.12 & 50.0 & 1.5 & 47 \\
\hline Range & & $575.2-712.2$ & $44.8-142.8$ & $162-380$ & & $0.12-0.88$ & $0.0-72.0$ & $1.1-1.5$ & $43-58$ \\
\hline Mean & & 654.5 & 105.1 & 241 & & 0.54 & 44.8 & 1.4 & 47.8 \\
\hline EKP III & \multicolumn{9}{|c|}{$\mathrm{N}^{\circ} 5^{\circ} 18^{\prime} 56.8^{\prime \prime} ; \mathrm{E008}^{\circ} 13^{\prime} 25.1^{\prime \prime} ; 82 \mathrm{~m}$ ASL } \\
\hline Ap & $0-15$ & 754.8 & 123.6 & 150 & SL & 0.82 & 40.9 & 1.1 & 62 \\
\hline $\mathrm{Bt} 1$ & $15-25$ & 619.6 & 120.4 & 260 & SCL & 0.46 & 67.4 & 1.4 & 47 \\
\hline $\mathrm{Bt} 2$ & $25-42$ & 735.2 & 64.8 & 200 & SL & 0.32 & 0.0 & 1.5 & 43 \\
\hline Range & & $619.6-754.8$ & $64.8-123.6$ & $150-260$ & & $0.32-0.82$ & $0.0-67.4$ & $1.1-1.5$ & $43-62$ \\
\hline Mean & & 703.2 & 102.9 & 203 & & 0.53 & 36.1 & 1.3 & 51 \\
\hline
\end{tabular}


Table.6: Chemical properties of the soil mapping unit

\begin{tabular}{|c|c|c|c|c|c|c|c|c|c|c|c|c|c|c|c|}
\hline Horizon & $\begin{array}{l}\text { Depth } \\
\text { (cm) }\end{array}$ & $\begin{array}{l}\mathrm{pH} \\
\left(\mathrm{H}_{2} \mathrm{O}\right)\end{array}$ & $\begin{array}{l}\text { pH } \\
\text { ( KCl) }\end{array}$ & $\Delta \mathrm{pH}$ & $\begin{array}{l}\text { Org.C } \\
(\%)\end{array}$ & $\mathbf{T N}(\%)$ & $\begin{array}{l}\mathbf{C :} \\
\mathbf{N}\end{array}$ & $\begin{array}{l}\text { Avail.P } \\
\left(\text { mgkg }^{-1}\right)\end{array}$ & $\mathbf{C a}$ & Mg & $\begin{array}{l}\text { Exchangeable } \\
\text { K }\end{array}$ & $\mathbf{N a}$ & $\begin{array}{l}\text { Exchangeable } \\
\left(\mathrm{Al}^{3+}+\mathrm{H}^{+}\right)\end{array}$ & ECEC & BS \% \\
\hline EKP I & \multicolumn{15}{|c|}{$\mathrm{N05}^{\circ} 19 ’ 16.84 " ; \mathrm{E008}^{\circ} 13$ ' 36.1" ; $108 \mathrm{~m} \mathrm{ASL)}$} \\
\hline Ap & $0-17$ & 5.0 & 4.9 & -0.1 & 0.60 & 0.04 & 15 & 20.6 & 2.2 & 1.40 & 0.14 & 0.48 & 0.9 & 5.12 & 82.0 \\
\hline Bw1 & $17-62$ & 5.6 & 4.4 & -1.2 & 0.74 & 0.06 & 12 & 17.3 & 1.6 & 1.00 & 0.10 & 0.08 & 1.6 & 4.34 & 64.0 \\
\hline Bw2 & $62-122$ & 5.1 & 4.8 & -0.3 & 0.50 & 0.03 & 17 & 20.8 & 2.6 & 1.50 & 0.37 & 0.37 & 1.7 & 6.54 & 74.0 \\
\hline $\mathrm{Crt}$ & $122-200$ & 5.6 & 4.7 & -0.9 & 0.40 & 0.03 & 13 & 15.4 & 1.2 & 0.80 & 0.10 & 0.08 & 1.6 & 3.74 & 58.0 \\
\hline Range & & $\begin{array}{l}5.0- \\
5.6\end{array}$ & $4.4-4.8$ & $0.1-0.9$ & $\begin{array}{l}0.40- \\
0.74\end{array}$ & $\begin{array}{l}0.03- \\
0.06\end{array}$ & $\begin{array}{l}12- \\
17\end{array}$ & $15.4-20.6$ & $\begin{array}{l}1.2- \\
2.6\end{array}$ & $\begin{array}{l}0.50- \\
1.50\end{array}$ & $0.10-0.37$ & $\begin{array}{l}0.08- \\
0.48\end{array}$ & $0.9-1.7$ & $\begin{array}{l}3.74- \\
6.54\end{array}$ & $\begin{array}{l}58.0- \\
74.0\end{array}$ \\
\hline Mean & & 5.3 & 4.7 & -0.6 & 0.56 & 0.18 & 14 & 18.5 & 1.9 & 1.18 & 0.18 & 0.25 & 1.4 & 4.94 & 69.5 \\
\hline EKP II & \multicolumn{15}{|c|}{$\mathrm{N05}^{\circ} 19^{\prime} 11.3^{\prime \prime} ; \mathrm{EO08}^{\circ} 13^{\prime} 36.8^{\prime \prime} ; 102$ m ASL } \\
\hline Ap & $0-8$ & 4.8 & 4.3 & -0.5 & 0.60 & 0.03 & 20 & 24.7 & 3.0 & 1.5 & 0.46 & 0.47 & 1.7 & 7.10 & 76.0 \\
\hline $\mathrm{Bt} 1$ & $8-53$ & 4.9 & 4.1 & -0.8 & 0.63 & 0.05 & 13 & 11.4 & 1.4 & 1.0 & 0.09 & 0.07 & 1.8 & 4.40 & 59.0 \\
\hline $\mathrm{Bt} 2$ & $53-114$ & 4.1 & 4.0 & -0.1 & 0.30 & 0.04 & 15 & 23.8 & 3.3 & 1.5 & 0.44 & 0.47 & 0.8 & 6.50 & 44.0 \\
\hline $\mathrm{Crt}$ & $114-200$ & 5.5 & 4.5 & -1.0 & 0.36 & 0.02 & 18 & 14.2 & 1.4 & 0.8 & 0.09 & 0.08 & 2.3 & 4.70 & 51.0 \\
\hline Range & & $\begin{array}{l}4.8- \\
5.5\end{array}$ & $4.1-4.5$ & $0.1-1.0$ & $\begin{array}{l}0.30- \\
0.63\end{array}$ & $\begin{array}{l}0.02- \\
0.05\end{array}$ & $\begin{array}{l}13- \\
20\end{array}$ & $11.4-24.7$ & $\begin{array}{l}1.4- \\
3.3\end{array}$ & $0.8-1.5$ & $0.09-0.44$ & $\begin{array}{l}0.07- \\
0.47\end{array}$ & $0.8-1.8$ & $\begin{array}{l}4.40- \\
7.10\end{array}$ & $\begin{array}{l}44.0- \\
76.0\end{array}$ \\
\hline Mean & & 4.8 & 4.2 & -0.6 & 0.47 & 0.04 & 17 & 18.5 & 2.3 & 1.2 & 0.27 & 0.27 & 1.7 & 5.70 & 58.0 \\
\hline EKP III & \multicolumn{15}{|c|}{${\mathrm{N} 05^{\circ}}^{18} 8^{\prime} 56.8^{\prime \prime} ;{\mathrm{E} 008^{\circ}}^{13} 3^{\prime} 25.1^{\prime \prime} ; 82 \mathrm{~m} \mathrm{ASL}$} \\
\hline Ap & $0-15$ & 4.9 & 4.1 & -0.8 & 0.2 & 0.01 & 20 & 16.5 & 1.0 & 0.8 & 0.09 & 0.07 & 2.2 & 4.20 & 47.0 \\
\hline Bt1 & $15-25$ & 4.4 & 4.2 & -0.2 & 0.4 & 0.03 & 13 & 13.2 & 2.3 & 1.7 & 0.35 & 0.34 & 0.4 & 5.10 & 92.1 \\
\hline $\mathrm{Bt} 2$ & $25-42$ & 5.5 & 4.6 & -0.9 & 0.1 & 0.01 & 10 & 12.9 & 2.6 & 1.0 & 0.07 & 0.06 & 1.5 & 5.30 & 71.0 \\
\hline Range & & $\begin{array}{l}4.4- \\
5.5\end{array}$ & 4.1-4.6 & $0.2-0.9$ & $0.1-0.4$ & $0.1-0.03$ & $\begin{array}{l}10- \\
20\end{array}$ & $12.9-16.5$ & $\begin{array}{l}1.0- \\
2.6\end{array}$ & $0.8-1.7$ & $0.07-0.35$ & $\begin{array}{l}0.06- \\
0.34\end{array}$ & $0.4-2.2$ & $\begin{array}{l}4.20- \\
5.30\end{array}$ & $\begin{array}{l}47.0- \\
71.0\end{array}$ \\
\hline Mean & & 4.9 & 4.3 & -0.6 & 0.2 & 0.02 & 14 & 14.2 & 2.0 & 1.2 & 0.17 & 0.16 & 1.4 & 4.90 & 70.0 \\
\hline
\end{tabular}


The mean values of the carbon/nitrogen ratio for the soils were 14, 17 and 14 for EKP I, EKP II and EKP III respectively. The small or narrow $\mathrm{C}: \mathrm{N}(<25)$ (Paul andClark, 1989) will positively influence microbial activities to ensure rapid mineralization of organic matter with the consequent release of nutrient elements into the soil solution for crop plant assimilation (Akpan-Idiok et al.,2012) thus the carbon-nitrogen ratio of the soils falls within the given range. The available phosphorus content followed an irregular increase and decrease with depth in all the mapping units with the mean values of $18.5 \mathrm{mg} / \mathrm{kg}, 18.5$ $\mathrm{mg} / \mathrm{kg}$ and $14.2 \mathrm{mg} / \mathrm{kg}$ for EKP I, EKP II and EKP III respectively. Phosphorus content in soils was rated medium for mapping unit EKP I and EKP II and low for EKP III when compared with the critical value of $15 \mathrm{mg} / \mathrm{kg}$ (Enwezor et al., 1990 \& Adepetu, 2000).

The exchangeable bases of the all soil mapping units were low. Exchangeable $\mathrm{Ca}$ dominated the soil exchange site. A similar result was reported elsewhere (Fasina et al., 2006 \& Noma et al., 2004). Means of exchangeable acidity were $1.4 \mathrm{cmol} / \mathrm{kg}, 1.7 \mathrm{cmol} / \mathrm{kg}$ and 1.4 $\mathrm{cmol} / \mathrm{kg}$ for EKP I, EKP II and EKP III respectively. This showed that preponderance of exchangeable $\left(\mathrm{Al}^{3+}+\mathrm{H}^{+}\right)$ played a major role in soil acidity in the humid tropical soils of Ekpri Ibami. Effective cation exchange capacity values of the sois of Ekpri Ibami landscape were $4.94 \mathrm{cmol} / \mathrm{kg}$, $5.70 \mathrm{cmol} / \mathrm{kg}$ and $4.90 \mathrm{cmo} / \mathrm{kg}$ for EKP I, EKP II and EKP III respectively. The ECEC were generally low as they fall within the critical value of $(<8 \mathrm{cmol} / \mathrm{kg})$ as provided by FAO, (1976) for soils of the ecological zone. This shows that the soils at their natural $\mathrm{pH}$ remain low in cation exchange and has low ability to retain nutrients (Yakubu, 2006). The means base saturation of the different mapping units were $69.5 \%, 58.0 \%$ and $70.0 \%$ for EKP I, EKP II, EKP III respectively. A similar result was obtained by Abua and Eyo (2013) and Bulktrade (1989)in soils of Akamkpa. Meanwhile, according to FAO (1999) base saturation > $50 \%$ is regarded as fertile and rated high while $<50 \%$ are regarded as not fertile soil and rated low. With the high base saturation, the basic nutrients must have occurred in available forms in soil solution in spite of the low cation reserves in the soil.

\subsection{Soil classification}

The soil morphological, physical and chemical properties were used to classify the soil unit according to USDA (2010). The mapping units identified at Ekpri Ibami landscape proposed for Oil palm production and denoted by EKP I, EKP II and EKP III and discussed as follows:
EKP I: This group of soil showed a moderate level of weathering and lacks the extensive amount of clay accumulation. They were identified with a weak horizon and with the presence of a cambic diagnostic horizon along with an ochric epipedon and moist colour value of 3 or more. Soils are developed on colluvial deposits. The soil is characterized with a weak coarse granular structure at the surface, improperly drained down the soil depth during the rains and are placed in the order of Inceptisols. With udic moisture regime occurring within the study location, the soil fits into the Udepts suborder and with a base saturation of $50 \%$ in one or more horizons at a depth between 25 and 75 $\mathrm{cm}$, they are further placed into Eutrudepts great group. The soils were further classified as Arenic Eutrudept at subgroup category due to the irregular decrease in organiccarbon content between a depth of $25 \mathrm{~cm}$ and at a depth of $125 \mathrm{~cm}$

EKP II: With the presence of argillic horizon and low base saturation less than $50 \%$ (with $\mathrm{NH}_{4} \mathrm{OAc}$ ) in with the depths 20 and $100 \mathrm{~cm}$ from the surface mineral soils. Thus qualified to be placed in order Ultisol and great group Hapludult and subgroup Typic Hapludult, according to USDA Soil Taxonomy classification.

EKP III: with increasing depth, do not have a clay decrease of 20 percent or more (relative) from the maximum clay content and so fit into Paleudults at the great group level and at subgroup level classified as Aquic Paleudults.

\subsection{Land evaluation for the proposed oil palm production}

3.4.1 Land qualities and land use requirement for oil palm production

\subsubsection{Climate (c)}

Climate parameters considered in the study were annual rainfall, mean annual temperature, and relative humidity. In Akamkpa LGA of Cross River State rainfall is a sufficient factor for oil palm production and has a mean annual rainfall of $2000 \mathrm{~mm}$ (NIMET, 2015). The mean annual temperature and relative humidity in this region are greater than $25^{\circ} \mathrm{C}$ and $80 \%$ respectively as shown in Table (7). The climatic characteristics were rated $100 \%$ since they exceed $2000 \mathrm{~mm}, 25^{\circ} \mathrm{C}$ and $75 \%$ provided by Sys (1985) for S1.

\subsubsection{Topography (t)}

The topography of the study area was on strongly undulating landscapeswith EKP I mapping unit occurring on relatively flat terrain (0-8 \%), crest region. EKP II and EKP III mapping units were located at a convexly shaped angle with gradient range of $8-16 \%$. According to the criteria set by Sys (1985) for oil palm production EKP I is 
rated highly suitable (S1) while EKP II and EKP III are moderately suitable (S2) (Table ). But, soils of EKP III may pose harvest and transportation challenges. Contour farming and strip cropping should be adopted along the slope gradient with reduced grazing and removal of vegetation. This practice will check erosion in EKP II mapping unit.

Table.7: Suitability class scores of the pedons Oil palm cultivation

\begin{tabular}{llll}
\hline Parameters & EKP I & EKP II & EKP III \\
\hline Climate (c) & & & \\
\hline Annual Rainfall $(\mathrm{mm})$ & $\mathrm{S} 1(100)$ & $\mathrm{S} 1(100)$ & $\mathrm{S} 1(100)$ \\
\hline MAT $\left({ }^{\circ} \mathrm{C}\right)$ & $\mathrm{S} 1(100)$ & $\mathrm{S} 1(100)$ & $\mathrm{S} 1(100)$ \\
\hline Relative humidity $(\%)$ & $\mathrm{S} 1(100)$ & $\mathrm{S} 1(100)$ & $\mathrm{S} 1(100)$ \\
\hline Topography(t) & & & \\
\hline Slope $(\%)$ & $\mathrm{S} 1(100)$ & $\mathrm{S} 2(65)$ & $\mathrm{S} 2(70)$ \\
\hline Wetness(w) & & & \\
\hline Flooding & $\mathrm{S} 1(100)$ & $\mathrm{S} 1(100)$ & $\mathrm{S} 3(49)$ \\
\hline Drainage & $\mathrm{S} 1(85)$ & $\mathrm{S} 1(85)$ & $\mathrm{N} 1(20)$ \\
\hline Soil Physical properties(s) & & & \\
\hline Texture & $\mathrm{S} 1(90)$ & $\mathrm{S} 2(50)$ & $\mathrm{S} 2(60)$ \\
\hline Structure & $\mathrm{S} 1(75)$ & $\mathrm{S} 1(75)$ & $\mathrm{S} 1(75)$ \\
\hline Depth(cm) & $\mathrm{S} 1(100)$ & $\mathrm{S} 1(100)$ & $\mathrm{S} 3(44)$ \\
\hline Fertility(f) & & & \\
\hline ECEC(cmol/kg) & $\mathrm{S} 3(47)$ & $\mathrm{S} 2(60)$ & $\mathrm{S} 3(44)$ \\
\hline Base Saturation $(\%)$ & $\mathrm{S} 1(80)$ & $\mathrm{S} 1(70)$ & $\mathrm{S} 1(90)$ \\
\hline pH(H $\left(\mathrm{H}_{2} \mathrm{O}\right)$ & $\mathrm{S} 2(55)$ & $\mathrm{S} 2(50)$ & $\mathrm{S} 3(40)$ \\
\hline OC(\%)(0-15 cm) & $\mathrm{S} 3(48)$ & $\mathrm{S} 3(45)$ & $\mathrm{S} 3(49)$ \\
\hline Aggregate Suitability & & \\
\hline Potential & $\mathrm{S} 3(38.1)$ & $\mathrm{S} 3(26.3)$ & $\mathrm{N} 2(10.9)$ \\
\hline Actual $(\mathrm{current})$ & $\mathrm{S} 3(37.3)$ & $\mathrm{N} 1(23.6)$ & $\mathrm{N} 2(9.9)$ \\
\hline & & & \\
\hline
\end{tabular}

\subsubsection{Wetness (w)}

In terms of soil wetness, the characteristics considered under this land quality group were flooding and drainage. They were no flooding problems in EKP I and EKP II mapping units as they were very well drained and had no characteristics of limiting drainage probably due to the sandy and gravelling properties of the soilsand were rated $100 \%$ (S1) for both flooding and drainage. But EKP III mapping unit is influenced by the inflow of water from the streams and rated between $25-49 \%$ (S3) for flooding and drainage.

\subsubsection{Soil physical properties (s)}

Soil physical properties considered were texture, structure and soil depth. Comparing the land qualities (Table 2). All the mapping units were rated between $40-90$ $\%$ for both soil texture and structure. But, soil texture is generally optimum for oil palm production with moderate to high suitability. EKP I = S1, EKP II = S2 and EKP III= S3.

\subsubsection{Soil fertility (f)}

The soils effective cation exchange capacity by summation, base saturation (BS) and organic carbon were evaluated as potential fertility characteristics they are not easily altered. The matching scores as shown in Table (6) showed that the ECEC values of the three mapping units were marginally suitable (S3) the criteria required for oil palm production as suggested by Sys (1985) with an average score of $49 \%$ while the base saturation is highly suitable (S1) with suitability scores between 80-100\%. The $\mathrm{pH}$ of the different mapping units was moderately suitable (S2). The organic carbon scores showed that the EKP I, EKP II and EKP III are moderately suitable (S2) and pose greater fertility challenge in the production of oil palm, so, requires the increase in organic matter content.

\subsection{Oil palm Suitability}

The aggregate scores, S3(38.1), S3 (26.3) and N2 (10.9) are potentially suitable scores for EKP I and EKP II and EKP III respectively. While S3 (37.3), N1 (23.6), N2 (9.9) are actual (current) suitable scores. Table (8) presents the non-parametric and parametric rating of the different soil mapping units.

Potential Suitability: EKP I and EKP II are marginally suitable for oil palm production and must, need continuous conservation and crop management practices such as appropriate and adequate fertilizer application, contouring, 
minimum tillage etc to ensure increased yield while conserving the soil nutrient level while EKP III is not suitable.

Actual (Current) Suitability: the aggregate scores showed that EKP I is marginally suitable while EKP II and EKP III are not suitable.

Table.8: Suitability classification of the mapping units

\begin{tabular}{|c|c|c|c|c|}
\hline $\begin{array}{l}\text { Mappi } \\
\text { ng }\end{array}$ & Potential & & $\begin{array}{c}\text { Curren } \\
\mathbf{t}\end{array}$ & \\
\hline & $\begin{array}{l}\text { Nonparame } \\
\text { tric }\end{array}$ & $\begin{array}{l}\text { Paramet } \\
\text { ric }\end{array}$ & $\begin{array}{l}\text { Non } \\
\text { paramet } \\
\text { ric }\end{array}$ & $\begin{array}{l}\text { paramet } \\
\text { ric }\end{array}$ \\
\hline EKP I & S2f & $\begin{array}{l}\mathrm{S} 3(38.1 \\
)\end{array}$ & S2f & $\begin{array}{l}\mathrm{S} 3(37.3 \\
)\end{array}$ \\
\hline $\begin{array}{l}\text { EKP } \\
\text { II }\end{array}$ & S3fs & S3(26.3 & $\mathrm{N} 2 \mathrm{tfs}$ & $\begin{array}{l}\mathrm{N} 1(23.6 \\
)\end{array}$ \\
\hline $\begin{array}{l}\text { EKP } \\
\text { III }\end{array}$ & $\mathrm{N} 2 \mathrm{wf}$ & $\begin{array}{l}\mathrm{N} 2(10.9 \\
)\end{array}$ & $\mathrm{N} 2 \mathrm{wf}$ & N2(9.9) \\
\hline
\end{tabular}

$\mathrm{f}=$ fertility limitation; $\mathrm{w}=$ wetnes $\mathrm{s}$ limitation; $\mathrm{s}=$ soil physical characteristic limitation; $\mathrm{t}=$ topography

3.6 Soil management practices for Oil palm
production at Ekpri Ibami
The soils of Ekpri Ibami in Akamkpa Local
Government Area of Cross River represented with mapping units EKP I, EKP II and EKP III were generally good for the agricultural purpose. But, their specific limitations have placed them into different suitability classes. The general management practices are as follows;

\subsubsection{Maintenance of soil fertility}

The basic cations such as $\mathrm{Ca}, \mathrm{Mg}$ and $\mathrm{K}$ appear to be very low in the soils of the study area due to high rainfall resulting into leaching of these cations out of soil solum (Esu, 2005). So, the proper soil management strategist to conserve or improve the fertility may include spreading of crop residues on the soils after harvesting, the inclusion of grasses and legumes during fallow.

\subsubsection{Adoption of conservation practices}

For best production; it is recommended that the practice of annual burning of bushes and plant residues at the start of farming, extensive grazing of the bushes by livestock and the felling of trees be discouraged while contour farming and strip cropping along slopes be encouraged to reduce the speed of runoff and subsequently reduce erosion and leaching in the area.

\subsubsection{Organic manures and chemical fertilizer application}

Oil palm requires balanced and the sufficient amount of micro and micronutrients for production. The study shows that the soil mapping units are generally low in soil basic nutrient yielding marginally to moderate suitability. However, to ameliorate the nutrient status of the soil adequate application of fertilizer in a split dosage at 3 months interval should be adopted (FDDD, 1989). Farmyard manure (FYM) of 75 to $100 \mathrm{~kg}$ or 90 to $100 \mathrm{~kg}$ of green manure and $5 \mathrm{~kg}$ neem cake should be added per each oil palm tree along the second dose of fertilizer. The practice will also reduce soil water erosion in the study area and will return the much-needed plant nutrient element to the soil (Esu, 2005; Onyekwere etal., 2001; Akpan-idiok, 2012).

\section{CONCLUSION}

Three mapping units (EKP I, EKP II and EKP III) derived from basement complex parent material of Akamkpa Local Government Area were mapped, characterized, classified and evaluated for its suitability for oil palm cultivation. The soils were strong to moderately acidic (4.8 to 5.3). The soils also have low inherent of natural fertility with low organic carbon content, total nitrogen, and moderately available phosphorus. Low effective cation exchange capacity and high base saturation which may have occurred in available forms in solutions in spite of the low cation reserves in the soil.

Parametrically, mapping units EKP I and EKP II were marginally suitable and EKP III not suitable for the proposed oil palm production. Non-parametrically, EKPI is moderately suitable, EKP II is marginally suitable and EKP III is not suitable, for growing oil palm. The prevailing limitations on the Ekpri Ibami landscape for oil palm production include fertility, wetness, and topography and soil physical properties. Fertility factor happens to be the most limiting factor in all the mapping units. And thus can be ameliorated through the application of organic manures, NPK fertilizer and liming may also improve the soil fertility status. At EKP III which is seen to be limited by wetness 
and drainage, proper drainage channels should be constructed since the proposed tree crop requires welldrained soil to encourage microbial biomass. Also, waterlogging crops can be grown in the area.

There is no declaration of conflict of Interest.

\section{REFERENCES}

[1] Abua, M.\& Eyo E. (2013). Assessment of soils around quarry terrain in Akamkpa local government area, Cross River State-Nigeria. Merit Research Journal of Agricultural Science and Soil Sciences, 1(1), 001-005.

[2] Adepetu, J.A. (2000). Interpretation of soil test data In: Simple soil, Water and Plant Testing Technologies for Soil Resource Management. IITA Ibadan/FAO Rome, pp. 89 - 97.

[3] Afangide, A. I., E. O. Francis \& E. I. Eja (2010). A Preliminary investigation into the selected towns in parts of South Eastern Nigeria. Journal of Sustainable Development, 3(3), 275-282.

[4] Aki, E. E, I. E. Esu \& Akpan-Idiok, A. U. (2014) Pedological Study of Soils Developed on BiotiteHornblende- Gneiss in Akamkpa Local Government Area in Cross River State, Nigeria.International Journal of Agricultural Research, 9, 187-199.

[5] Akpan-Idiok, A. U \& Ukwang, E.E. (2012). Characterization and Classification of Coastal Plain Soils in Calabar, Nigeria.Journal Agricultural Biotechnology and Ecology 5(3), 19-33.

[6] Attoe, E. E., Undie, U.I., \& Kekong, M. A. (2016). Evaluation of Alluvial and Upland Soils of Obubra Local Government Area of Cross River State, Nigeria for Okra (Abelmoschus Esculentus) Production. European Journal of Academic Essays 3(2):100-104

[7] Bulk \& Investment Co. Ltd. 1989. Soil and Land Use Survey of Cross River State, Main Report, Ministry of Agriculture and Natural Resources, Calabar, C.R.S, Nigeria. Pp 1-164.

[8] Dengiz, O., Mustafa, S., Esra, F., Saygin, F \&Atasoy, C. (2012). Morphological and physicochemical characteristics and classification of vertisol developed on Deltaic plain, Turkey. Open Journal of Soil Science, 2, 20-27.

[9] Dobos, E., Carre, F., Hengl, T., Reuter, H\& Toth G (2006). Digital soil mapping as support to the production of functional maps. Office for official publications of the European Communities, Luxembourg. EUR 22123 EN, 68p.
[10] Ekwueme, B. N. (1990). Ages and Petrologic Features of Precambrian Rocks from Oban Massif Southeastern Nigeria, Precambrian Resources 47:271-286.

[11] Enwenzor, W. O.; Udo, E. J.; Usoroh, N. J.; Ayotade, K. A.; Adepetu, J. A.; Chude, V. O. \& Udegbe, C. I. (1989). Fertilizer use and management practices for crops in Nigeria Series, 2: 63-64.

[12] Enwezor, W. O. E. J. \& R. A. Sobulo (1990). Fertility Status and productivity of the "Acid Sand" In: Udo, E. J., and Sobulo, R. A. (eds) Acid Sands of southern Nigeria. Soil Science. Social. Nigeria Special Monograph 1:56- 73.

[13] Esu, I. E. (2010). Soil characterization, Classification and survey. Heinneman Educational Books Publishers, Nigeria. 232pp.

[14] Esu, I. E. (2004). Soil Characterization and Mapping for food security and sustainable Environment in Nigeria, A keynote address presented at the 29th Annual Conference of the Soil Science Society of Nigeria, held at the University of Agriculture, Abeokuta, Ogun State.

[15] Esu, I. E. (2005). Characterization, Classification and Management Problems of the major soil orders in Nigeria. $26^{\text {th }}$ Inaugural lecture held at the University of Calabar, Calabar. ISBN 978-007-148-2. Pp 1-66.

[16] FDALR (1990). The Reconnaissance Soil Survey of Nigeria (1:650,000). Soils report vol. 4 (Anambra, Akwa Ibom, Benue, Cross River, Imo and Rivers). Federal Department of Agricultural Land Resources, Lagos. 375pp.

[17] Food and Agriculture Organization (FAO). (1976). A framework for land evaluation. Soils Bulletin 32. 64p.

[18] Food and Agriculture Organization (FAO) (1999). World Reference Base for Soil Resources. World Soil Resources Rep. Vol. 84. Food and Agricultural Organisation of the UN, Rome, Italy, 161p.

[19] FitzPatrick, E. A. (1986). Soils, Their Formation, Classification, and Distribution. Longman and Technical, 351pp.

[20] Gee, G. W \& Bauder, J. W. (1986). Particle size analysis. In Klute, A. (ed) Methods of soil analysis, part 1. Agronomy, Vol. 9. Am. Soc. Agron., Madison, W1 383- 411pp.

[21] Ibanga, I. J. (2003) Guidelines for soil survey classification and land use. De-Rio press Nig. Ltd 1 $408 \mathrm{pp}$

[22] IITA (2000) Selected methods for soil and plant analysis. International Institute of Tropical Agriculture 
manual series No.1 Review editions. IITA Ibadan, Nigeria 70pp.

[23] McBratney, A. B. Mendonça-SantoS, M.L. \& Minasny, B. (2003). Digital soil mapping. Geoderma, 117, 3-52.

[24] McKenzie,N.J. \& Ryan, P.J. (1999). Spatial prediction of soil properties using environmental correlation. Geoderma 89: 67-94.

[25] NMA (2015). Nigeria Meteological Agency, Calabar Station.

[26] Ofem, K. I, A. U. Akpan-Idiok, S. M. Afu \& I. E. Esu (2016). Land suitability evaluation of

a. residual and colluvial-alluvial soils for oil palm cultivation in Biase, Cross River State, Nigeria. African Journal of Agricultural Science and Technology (AJAST), 4,4, 683-691.

[27] Ogunkunle, A. O. (1993). Soils in land evaluation: an example with oil palm in Nigeria Soil Use and Management, 9(1), 35-40.

[28] Ogunkunle A.O. (2005). Soil Survey and Sustainable Land Management. Invited paper at the $29^{\text {th }}$ annual conf. of SSSN held at the University of Nigeria, Abeokuta, from 6th to 10th Dec. 2004.

[29] Onyekwere, I.N., Akpan-Idiok, A.U., Amalu, U. C., Asawalam, D.O. and Eze, P. C (2001). Constraints and Opportunities in Agricultural Utilization of some Wetland Soils in Akwa Ibom State. (Presented at the $27^{\text {th }}$ Annual Conference of the Soil Science Society of Nigeria held at Calabar - Nigeria). Pp 139 - 149

[30] Orimoloye, J., I. K. Ugwa \& S. O. Idoko (2010).Soil management strategies for rubber cultivation in an undulating topography of Northern Cross River State. Journal of Soil Science and Environmental management. 1(2), 34- 39.

[31] Soil Survey Staff (2002). Field Book for describing and sampling soils. Version 2.0 National Soil Survey Center, Natural Resources Conservation Service, USDA Lincoln Nebraska.

[32] Soil Survey Staff, (2010). Keys to Soil Taxonomy (pp338) $11^{\text {th }}$ Edition Government Printing Office, Washington, DC, ISBN: 9780160854279.

[33] Sys, C. (1985). Land evaluation. Ghent, Belgium: the State University of Ghent, International Training Centre for post-graduate soil scientists; Algemeen Bestuur van de Ontwikkelingssamenwerking.

[34] Sys, C., Van Ranst, E. \& Debaveye, J. (1991). Land evaluation. Part 2: Methods in land evaluation. Agricultural publications 72. General Administration of DevelopmentCooperation of Belgium, Brussels. $247 \mathrm{p}$.

[35] Udo E. J., Ibia T.O., Ogunwale J.A., Ano A.A. \& Esu I. E.(2009). Manual of soil, plant and water analysis (pp.82-92.). Sibon Book Limited, Lagos.

[36] Udoh, B. T., Ogunkunle, A. O. \& Olaleye, A. O. (2006). Land suitability evaluation forbanana/plantain (Musa spp.) cultivation in Akwa Ibom State of Nigeria. Journal of Research in Agriculture, 3(3), 1-6. 